\title{
Psychological Interventions for Irritable Bowel Syndrome: A Meta-Analysis of Iranian Randomized Trials
}

\author{
Gholamreza Jandaghi, PhD'; Ali Zia-Tohidi, MScㄹ; Manijeh Firoozi, PhD²* \\ 'Faculty of Management and Accounting, Farabi Campus, University of Tehran, Qom, Iran \\ ${ }^{2}$ Department of Psychology, Faculty of Psychology and Educational Sciences, University of Tehran, Tehran, Iran
}

\begin{abstract}
Background: : Irritable bowel syndrome (IBS) is a common health problem, with considerable effects on the individual's quality of life, mental health, work productivity, and financial aspects. Psychological interventions, which are commonly used as adjunct treatments, have received fairly strong empirical support. In Iran, several randomized clinical trials (RCTs) have evaluated the efficacy of these treatments; however, the results of these RCTs have not been synthesized yet. Therefore, in this meta-analysis, we aimed to summarize the results of these trials on the efficacy of psychological interventions in Iranian adults with IBS.

Methods: We searched IranDoc, ElmNet, PubMed, and Scopus for eligible RCTs. The risk of bias was assessed in each trial, according to the Cochrane guidelines, and the random-effect model was used to pool the effect size (EF) across trials.

Results: Twenty RCTs met the eligibility criteria and were included in the meta-analysis. Compared to the controls, the standardized mean difference (SMD) for psychological interventions was large regarding the severity of IBS symptoms $(-1.21 ; 95 \% \mathrm{Cl}:-1.63$ to $-0.80)$, anxiety (-0.97; $95 \% \mathrm{Cl}:-1.32$ to -0.63$)$, and depression $(-0.86 ; 95 \% \mathrm{Cl}:-1.28$ to -0.44$)$. There was considerable heterogeneity among the included RCTs regarding all three outcomes, which could not be explained by the available information. On the other hand, the EF of health-related quality of life was 0.64 (95\% Cl: 0.38 to 0.85$)$, with no significant heterogeneity among RCTs.

Conclusion: The existing evidence suggests that psychological interventions can be highly effective in improving the severity of IBS symptoms, mental health, and quality of life for Iranian adults with IBS. However, some weaknesses should be considered in the interpretation of the results and future research. The risk of randomization was high or unclear in almost all of the existing trials; there was no single large trial in this area; and there was substantial inconsistency in the EFs, which might be related to methodological or clinical moderators.

Keywords: Iran, Irritable bowel syndrome, Meta-Analysis, Psychotherapy

Cite this article as: Jandaghi G, Zia-Tohidi A, Firoozi M. Psychological interventions for irritable bowel syndrom: a meta-analysis of iranian randomized trials. Arch Iran Med. 2021;24(6):496-504. doi: 10.34172/aim.2021.71
\end{abstract}

Received: September 19, 2019, Accepted: October 21, 2020, ePublished: June 1, 2021

\section{Introduction}

The gut and the brain have close and complex interactions in the human body. Therefore, any disturbance in these interactions can lead to functional gastrointestinal disorders (FGIDs). ${ }^{1}$ The most common FGID is irritable bowel syndrome (IBS), which affects about one-tenth of the population. ${ }^{2}$ In Iran, despite numerous studies, the precise prevalence of IBS is not clear, as previous studies have reported widely inconsistent results. ${ }^{3,4}$ However, the prevalence of this condition seems to be lower than the international pooled prevalence rate.

IBS is associated with lower quality of life and higher levels of anxiety and depression..$^{5-7}$ According to a study conducted in Iran, $75 \%$ of IBS patients were also diagnosed with at least one anxiety or depressive disorder. ${ }^{8}$ Overall, the relationship between IBS and anxiety/mood disorders is so strong that having anxiety or depression is considered an indicator of IBS. ${ }^{9}$ Despite all advances in science, the exact pathology of IBS remains unclear. Currently, the pathology of this disorder involves complex interactions between biological, psychological, and social factors. According to this biopsychosocial approach, which is widely agreed upon, genetics and environmental factors (i.e., early experiments, parental beliefs and behaviors, social learning, social support, culture, social stress, traumas, and infections) affect the brain and the gut, and therefore, disturb their interactions (through the hypothalamus-pituitary-adrenal axis and autonomic nervous systems), leading to functional gastrointestinal symptoms (see reference 10 for review).

Considering the role of psychological and social factors, psychological treatments seem to be effective for IBS. Today, substantial empirical evidence has been considered in treatment guidelines, especially for patients with refractory IBS, who do not respond well to pharmacological treatments ${ }^{11}$; have major anxiety or depression symptoms; or wish for such treatments. ${ }^{12,13}$ Although there is fairly strong empirical evidence regarding the efficacy of different psychological treatments for IBS, ${ }^{14-17}$ the results are mainly based on studies conducted in South America and Europe. Therefore, caution must be taken when generalizing these results to the Iranian 
population, as cultural differences may affect IBS through different physical (e.g., diet and environmental hygiene), psychological (e.g., disease-related beliefs, anxiety, and somatization), and social (e.g., family systems, food, and digestion-related taboos) factors. ${ }^{18}$ Also, psychological treatments may have different effects on different populations with different cultural backgrounds. ${ }^{19}$

According to our preliminary search of Iranian scientific databases, several randomized clinical trials (RCTs) have assessed the effects of psychological interventions on various outcomes of IBS. Therefore, it seems necessary to synthesize the reported results, review the existing evidence, and try to identify the research gaps to be filled.

\section{Materials and Methods \\ Search Strategy}

This study was a systematic review and meta-analysis. We searched ElmNet, IranDoc, PubMed, and Scopus databases, using the following keywords (or their Persian equivalents): "Irritable bowel" in combination with "psycho»", "cogniti*”, "acceptance", "meaning", "mindfulness", "relaxation", "biopsychosocial", "rehabilitation", or "educat*". In our search of English databases, we limited the search to Iranian studies by using the following words: "Iran", "Iranian", and "Persian".

\section{Eligibility Criteria}

The inclusion criteria for the RCTs were as follows: having a sample of at least ten participants per group (at allocation); use of a psychological intervention (not merely education); conducting the trial on Iranian IBS patients; and lack of obvious inconsistencies or numerous errors in reports (e.g., multiple typos in the outcome scores). We included journal articles and theses in this meta-analysis and looked for possible reports in all included studies, as recommended by the Cochrane collaboration. ${ }^{20}$

\section{Risk of Bias Assessment}

Risk of bias was assessed, according to the Cochrane guidelines, ${ }^{21}$ except for blinding, as the psychotherapists could not be blinded to the treatment, and blinding the assessors was not relevant because of using self-reports.

\section{Outcomes}

Based on the available evidence, we considered four outcomes in this meta-analysis, namely, the severity of IBS symptoms, anxiety, depression, and quality of life.

\section{Data Preparation and Statistical Analysis}

We calculated the standardized mean difference (SMD) as the primary effect size (ES) measure, since the assessment tools in the included trials varied, limiting the possibility of using the raw mean difference (MD). However, since several trials had used similar scales, allowing the meta-analysis of raw MDs, we also reported the MDs and included the forest plots in supplementary file 1. To calculate ES, we divided the change scores (post-treatment/follow-up minus pre-test) by the pretest standard deviation (SD) to obtain more precise and less biased estimates of ES in small trials, as suggested by Moris. ${ }^{22}$ Also, Hedges' correction for small sample trials applied to all SMDs.

We checked the normality of data from each trial by assessing the distance of the mean from the minimum and maximum possible values. If the distance was less than $1 \mathrm{SD}$, it was considered as an indication of severe skewness. When skewness was detected, a sensitivity analysis was carried out to determine whether the study has considerable effects on the summary EF. In studies with multiple treatment arms, we combined the ESs to avoid the unit of analysis error and excessive weight on the trials (by repeating the control group), as the recommended method in the literature. ${ }^{23}$ However, in the subgroup analyses based on the treatment type, we could not use the combined ES. Therefore, we split the shared control group sample size to approximately equal samples and then included every treatment arm in the analysis as a separate trial (p510).

In a study by Shahbazi in 2013, rather than reporting the total score of IBS symptoms, two separate scores were reported for frequency and severity. Generally, to calculate the composite score, it is necessary to account for the correlation between scores, which was determined in a previous study introducing the measure. ${ }^{24}$ We used the random-effect model to estimate the pooled ES, allowing for heterogeneity. Moreover, the restricted maximum likelihood (REML) method was used to estimate $\tau^{2}$, as recent evidence suggests REML to be less biased than other estimators. ${ }^{25}$ Other indicators of heterogeneity were also determined in the present study $\left(\mathrm{I}^{2}, \mathrm{Q}\right.$, and significance level). If significant or substantial (even nonsignificant) heterogeneity was detected, we determined the outliers and moderators. As recommended previously, we pre-specified the potential moderators to avoid posthoc analyses, which are prone to type I error. ${ }^{26}$ We selected two potential moderators, namely, type of treatment and researcher's education $(\mathrm{MA} / \mathrm{MSc}$ or $\mathrm{PhD})$; the latter was selected, based on our previous meta-analysis, which revealed its significant effect. ${ }^{27}$

On the other hand, in this meta-analysis, we did not assess publication bias, since there is no appropriate test available to the best of our knowledge. The fail-safe $\mathrm{N}$ is not generally recommended due to its problems, ${ }^{28,29}$ and funnel plot (and its related tests) is only applicable if there are considerable differences between studies in terms of sample size, which is not the case in our study (see Jin et $\mathrm{a}^{29}$ for more details). All analyses were conducted in $\mathrm{R}$ software, using the Metafor package. ${ }^{30}$

\section{Results}

The literature search and screening resulted in 20 eligible RCTs reported in 30 articles/theses (Figure 1). The characteristics of these studies are presented in Table S1 (see Supplementary file 1). All included trials were 


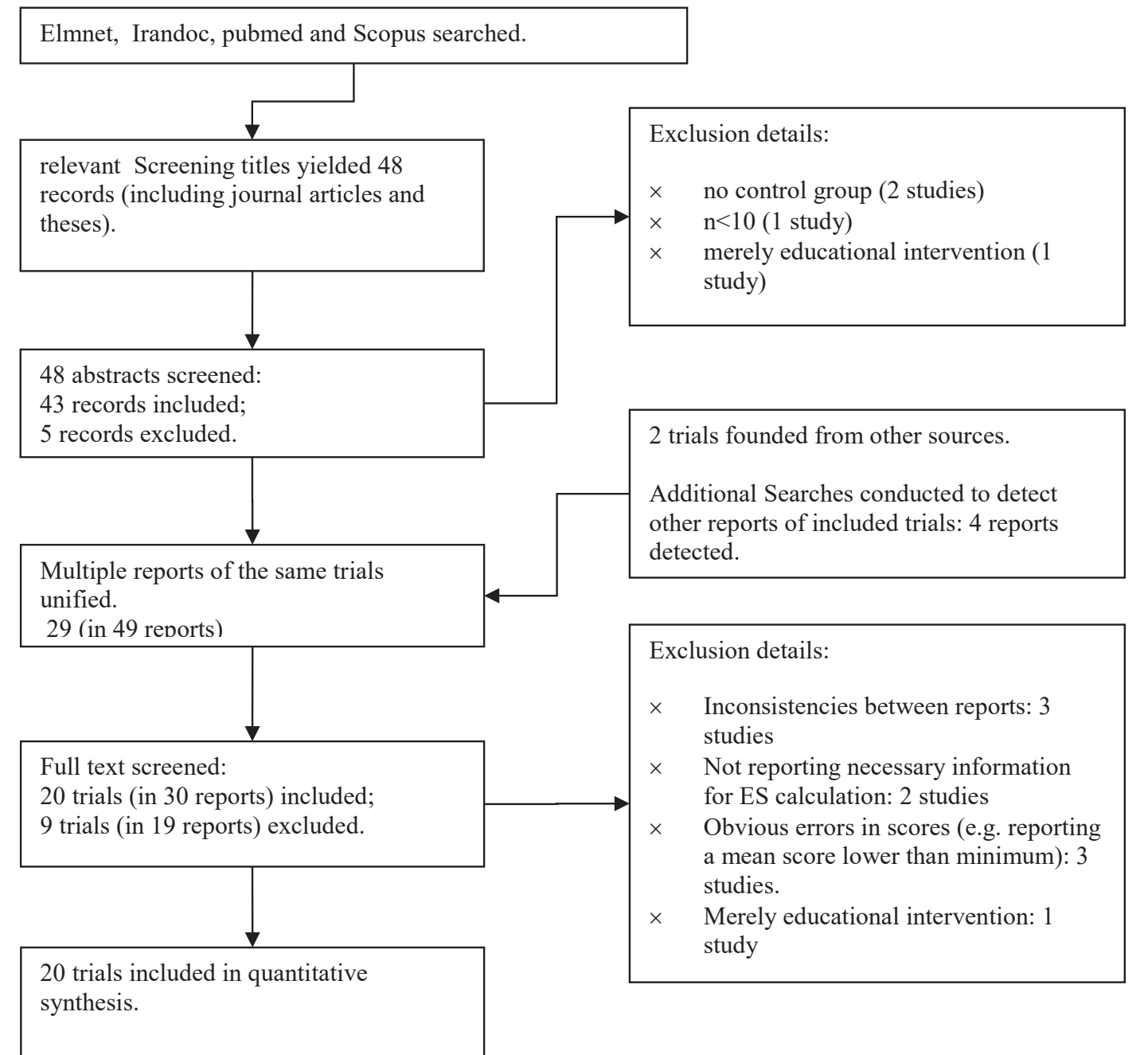

Figure 1. Flowchart of Screening Process.

conducted in the last twelve years, most had used groupbased therapy, and only eight of them had conducted follow-up assessments. The total sample included 781 patients (on average, 39 patients per trial). Four outcomes were reported frequently enough to allow meta-analysis: IBS symptom severity, anxiety, depression, and healthrelated quality of life (HR-QoL).

The results of the risk of bias assessments are provided in Supplementary Table 1 and the risks of bias across all included trials are presented in Figure 2. Only one study (5\%) was at low risk of bias for randomization processes (i.e., sequence generation and allocation concealment). The risk of incomplete outcome data was low in the majority of the included trials ( 12 trials, $60 \%$ ) and the risk of selective reporting was low in half of them ( 9 trials, $45 \%)$.

\section{IBS Symptom Severity}

Pooled ES from the ten studies that reported outcomes on IBS symptom severity was very large and significant (SMD $=-1.21 ; P<0.001)$. There was significant heterogeneity between trials $\left(\mathrm{Q}=28.9 ; P_{\mathrm{Q}}<0.001 ; \tau^{2}=0.3\right)$, which was mainly due to true heterogeneity rather than sampling error $\left(\mathrm{I}^{2}=68 \%\right)$. Additional investigations could not find any outliers and none of the potential moderators (i.e. treatment type, researchers' education level) could explain this heterogeneity. The Forest plot of this analysis is presented in Figure 3. Five trials used the IBS-symptom

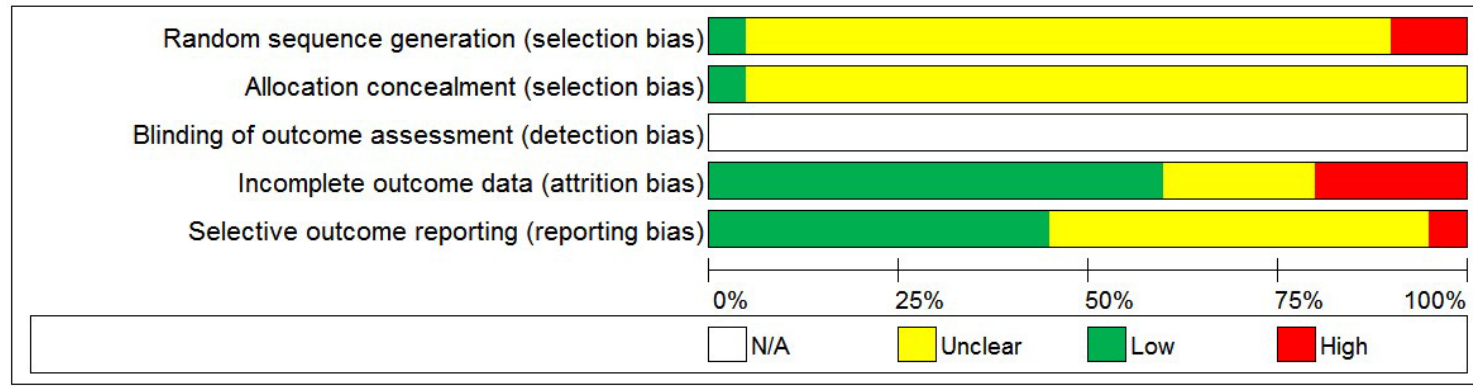

Figure 2. Summary Chart of the Risk of Bias Across Trials. 


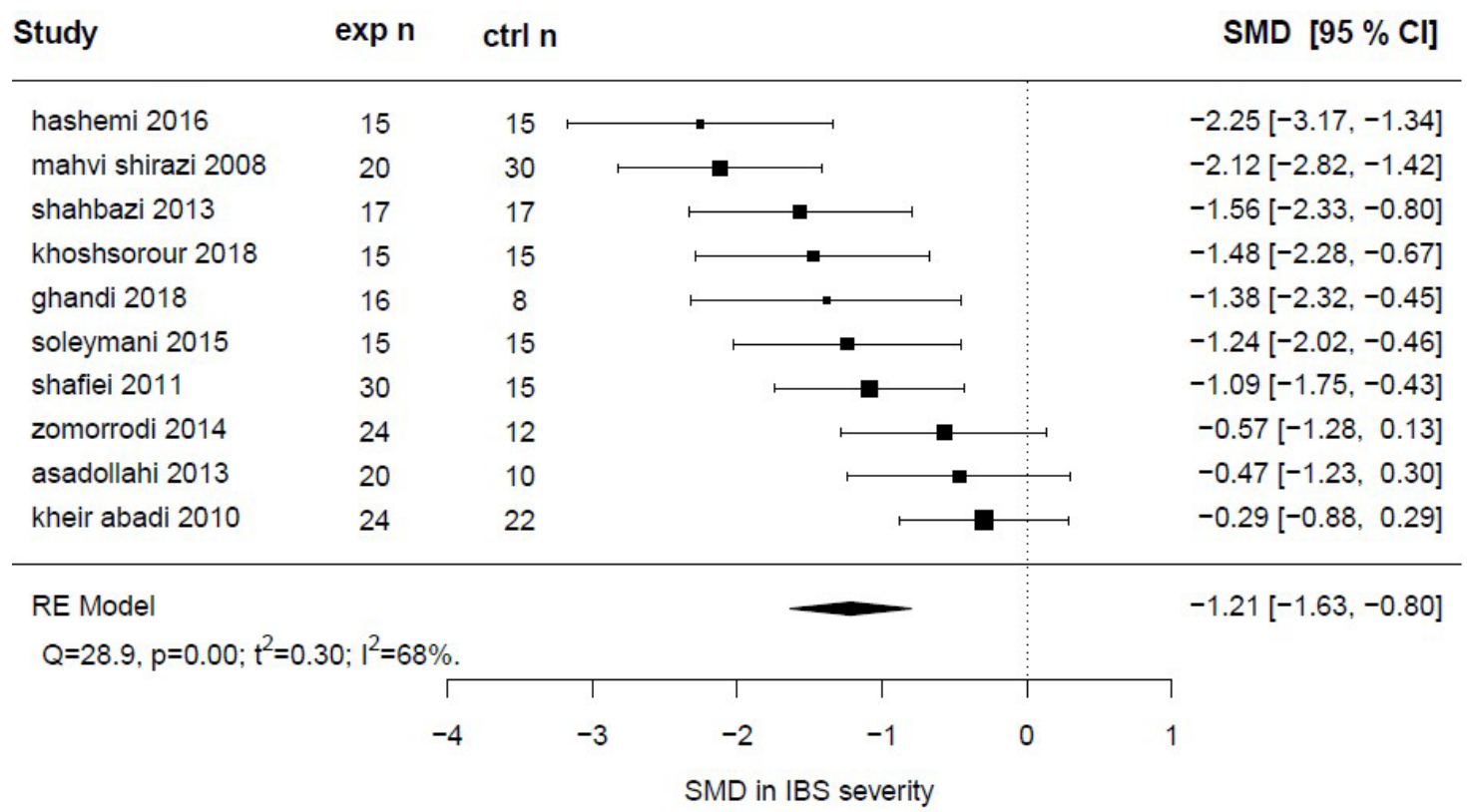

Figure 3. Forest Plot of the Standardized Mean Differences in IBS Symptom Severity at Post-treatment. RE, random effect; SMD, standardized mean difference.

severity scoring (IBS-SSS) scale which allowed us to calculate the raw MD, resulting in a MD of $-46.5(P=$ 0.003). Although there was considerable heterogeneity across these trials $\left(\mathrm{Q}=36.3, P_{Q}<0.001 ; \tau^{2}=876 ; \mathrm{I}^{2}=83 \%\right)$, the moderation analysis was not appropriate due to the small number of the included trials (the forest plot is presented in the Supplementary file 1).

At follow-up, analysis of five studies resulted in a significant homogeneous large ES (SMD = -1.09; $\left.P<0.001 ; \mathrm{Q}=5.41 ; P_{\mathrm{Q}}=0.25 ; \tau^{2}=0.01\right)$. Compared to post-treatment, there was a slight reduction in ES, but we speculated that this reduction might be due to excluding studies without follow-up assessments. So, we re-analyzed post-treatment effects, but only included these four studies which reported both post-treatment and followup scores. The results indicated that in these studies, there was no reduction in ES from post-treatment to follow-up, which supports our speculation.

Anxiety: One study reported pre-test mean scores that seemed to be practically impossible (mean anxiety scores of Beck's Anxiety Inventory [BAI] around 60 when the range of BAI scores is 13-63 and the cut point for severe anxiety is 26), so we excluded the study from the analysis. Pooled SMD from the eleven studies was unexpectedly large, with an unusual true heterogeneity index (SMD $\left.=-1.61, P=0.009 ; \tau^{2}=4.06\right)$. Additional investigations detected an outlier with an SMD of -8.55 . We checked the source again to make sure that the data had been extracted correctly and then removed it from the analysis, which reduced pooled SMD to $-0.97(P<0.001)$ and the $\tau^{2}$ to 0.19 , but the heterogeneity was still significant $(\mathrm{Q}=$ $\left.22.1, P_{\mathrm{Q}}=0.01, \mathrm{I}^{2}=61 \%\right)$. There were no other outliers and none of the moderators could explain the heterogeneity. The Forest plot is presented in Figure 4. Three studies had used BAI, which allowed us to calculate the raw MD, resulting in a mean difference of $-3.62(P=005)$, with non-significant but moderate heterogeneity $\left(\mathrm{Q}=3.8, P_{\mathrm{Q}}=\right.$ $0.15 ; \tau^{2}=1.93 ; \mathrm{I}^{2}=38 \%$; the forest plot is presented in the Supplementary file 1).

At follow-up, only three studies (see Table S1for details) reported scores on anxiety. Two out of three (Kamkar 2011 [skewed data] and Asadollahi 2013) had SMDs of about -0.5 and the third (Hazrati 2007) had a SMD of -4.3 .

\section{Depression}

Eight studies reported outcomes on depression which resulted in a large and significant pooled ES $(\mathrm{SMD}=-0.86$, $P<0.001)$. The studies were significantly heterogeneous $\left(\mathrm{Q}=20, P_{\mathrm{Q}}<0.01, \tau^{2}=0.24 ; \mathrm{I}^{2}=66 \%\right)$, but no outlier detected nor any of the potential moderators could explain this heterogeneity. The Forest plot is presented in Figure 5. The data in one study was severely skewed, but sensitivity analysis revealed no considerable influence on the summary effect. Three studies used Beck's Depression Inventory (BDI) with a homogeneous pooled raw $\mathrm{MD}$ of $-4.44\left(P=0.0008 ; \mathrm{Q}=1.42, P_{\mathrm{Q}}=0.49 ; \tau^{2}<0.01 ; \mathrm{I}^{2}=0 \%\right.$; the forest plot is presented in the Supplementary file 1).

Only three studies reported outcomes on depression, one with large ES (skewed data; SMD = -0.89), one with small (SMD $=-0.42)$, and one with no considerable effect $(\mathrm{SMD}=0.06)$.

\section{Health-Related Quality of Life}

Pooled ES from the nine studies which reported outcomes on HR-QoL was large and significant $(\mathrm{SMD}=0.89, P=$ $0.002)$, but heterogeneous $\left(\mathrm{Q}=34.62 ; P_{\mathrm{Q}}<0.001 ; \tau^{2}=\right.$ 0.63 ). There was one outlier, with an SMD of 3.44; after excluding this outlier, the SMD dropped to a mediumsized but homogenous effect $(\mathrm{SMD}=0.61, P<0.001$; $\mathrm{Q}$ $\left.=4.12, P_{\mathrm{Q}}=0.77 ; \tau^{2}=0.0 ; \mathrm{I}^{2}=0 \%\right)$. The Forest plot of this 


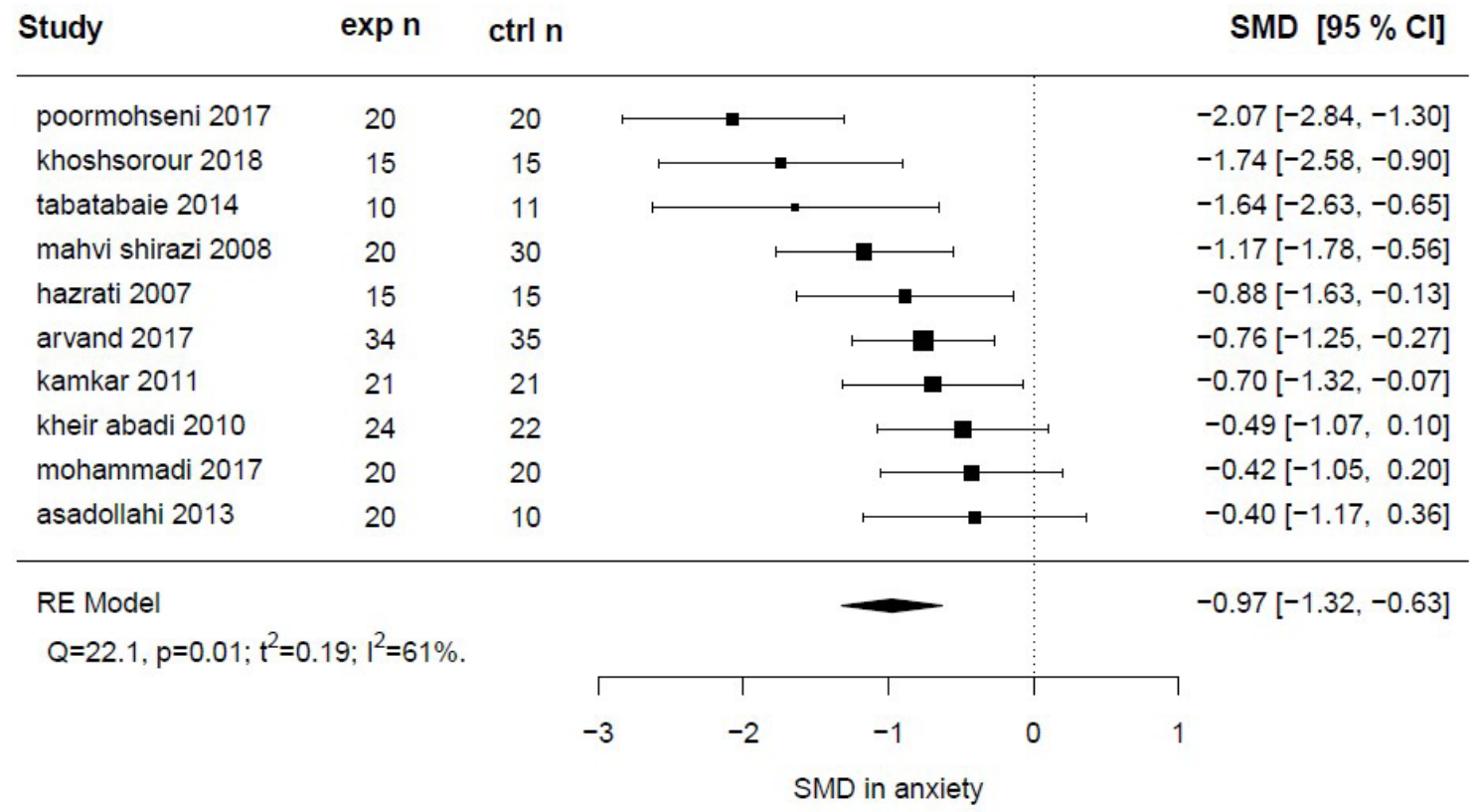

Figure 4. Forest Plot of the Standardized Mean Differences in Anxiety at Post-treatment. RE: random effect; SMD: standardized mean difference.

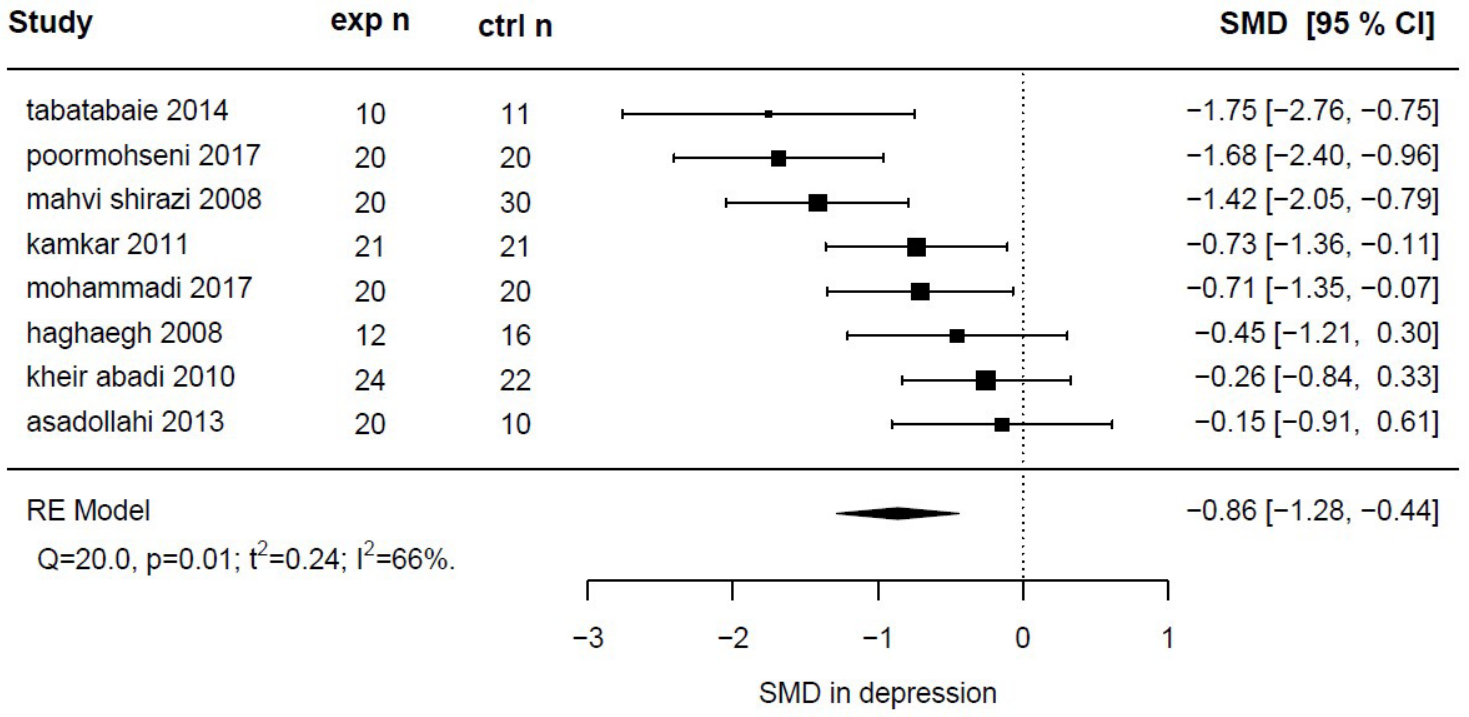

Figure 5. Forest Plot of Standardized Mean Difference in Depression at Post-treatment. RE: random effect; SMD: standardized mean difference.

analysis is presented in Figure 6. Six studies used the IBSQoL scale which resulted in a homogenous pooled raw $\mathrm{MD}$ of $-12.20\left(P<0.001, \mathrm{Q}=3.58, P_{\mathrm{Q}}=0.61 ; \tau^{2}=0 ; \mathrm{I}^{2}=\right.$ $0 \%$; the forest plot is presented in the Supplementary file $1)$.

At follow-up, pooled ES from four studies was unusually large but non-significant ( $\mathrm{SMD}=1.95, P=0.1$ ), with an unusual true heterogeneity index $\left(\tau^{2}=6.57\right)$, which was a function of one study with an SMD of 7.04 (Hazrati 2007). After checking the source for potential errors in data extraction, we removed the study from the analysis. After removing this study, pooled ES dropped to less than half, but became significant ( $\mathrm{SMD}=0.83, P<0.001)$, with no considerable heterogeneity $\left(\mathrm{Q}=3.47 ; P_{\mathrm{Q}}=0.32 ; \mathrm{I}^{2}=16 \%\right.$; $\left.\tau^{2}=0.03\right)$

\section{Discussion}

In Iran, several RCTs have been conducted to assess the efficacy of various psychotherapies for IBS. In the present study, we synthesized the results of these RCTs to gain a comprehensive view of the literature to help with treatment selection and future research. We can summarize the results of our meta-analysis as follows:

- On average, psychological interventions had large effects on the severity of IBS symptoms, anxiety, and depression.

- The present results suggested that trials had largely different ESs for the severity of IBS symptoms, 


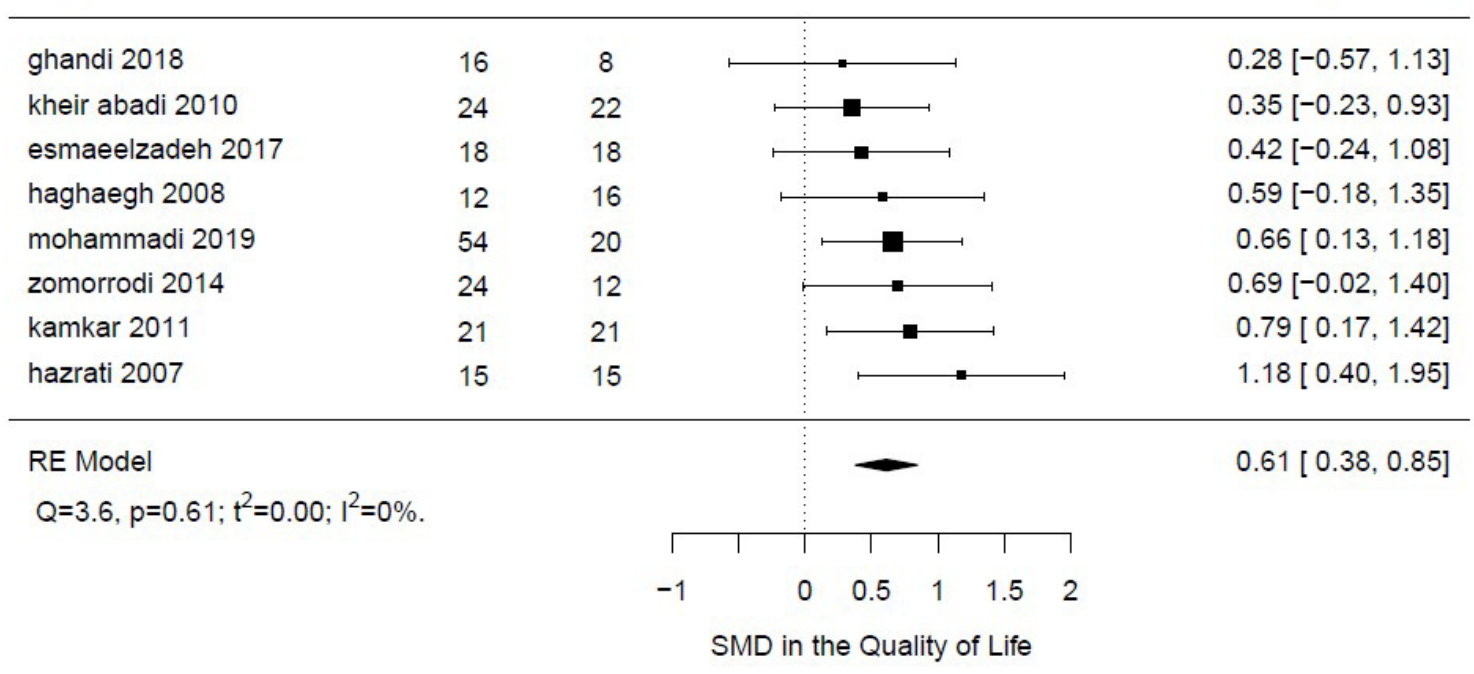

Figure 6. Forest Plot of the Standardized Mean Differences in Health-Related Quality of Life At Post-Treatment. RE, random effect.

anxiety, and depression, even after considering the treatment type. However, factors contributing to these differences are not clear, and methodological issues, therapists, and participants or a combination of these factors may be influential.

- Psychological interventions had medium effects on health-related quality of life, with no significant heterogeneity across trials, which is unlike other outcomes.

- In the follow-ups, although significant changes were observed in some outcomes, we cannot be confident about the results due to the small number of trials.

The results of this study are in line with the findings of previous meta-analyses, as psychological interventions had significant effects on various IBS outcomes; however, the ESs were considerably larger in our study. In a metaanalysis conducted by Laird and colleagues, ${ }^{31}$ the SMD of psychological interventions for the IBS symptom severity was -0.69 , which is much lower than our estimate. Moreover, Li and colleagues meta-analyzed the effect of cognitive-behavioral therapy on IBS and reported a pooled SMD of $-0.68 .{ }^{16}$ However, in their study, subgroup analysis revealed that the pooled SMD for trials with waitlist control groups (and not other active treatments) was much larger than their primary estimate, and more similar to our estimate in terms of magnitude $(\mathrm{SMD}=-1.21$ ).

Another meta-analysis on the efficacy of hypnotherapy indicated small ESs for the IBS symptom severity, anxiety, and depression $(\mathrm{SMD}<0.5)$ and a medium ES for QoL $(\mathrm{SMD}=0.56) .{ }^{15}$ However, the number of included studies was small (maximum of six trials).

The differences between our analysis of Iranian trials and other meta-analyses can be explained in different ways. The first simple explanation is to assume that the Iranian population responds better to psychological treatments. Nevertheless, there are other possibilities to be considered:

- The large ESs in the investigated trials could be a function of bias, as the randomization processes in all the included trials (except one study) were classified as having a high or an unclear risk of bias in both sequence generation and allocation concealment. There is also empirical evidence that studies with a high or an unclear risk of bias in these processes produce higher ESs (see Higgins and Altman ${ }^{21}$ ), especially when the outcomes are subjective. ${ }^{32}$

- Generally, studies with small sample sizes tend to produce larger ESs. The evaluated trials in our metaanalysis generally had sample sizes smaller than those included in the abovementioned meta-analyses. For instance, in a meta-analysis by Laird et al, $56 \%$ of the included studies had a sample size equal to or more than 50 patients, whereas this rate was $16 \%$ in our investigated trials. ${ }^{31}$

- Our meta-analysis included trials with wait-list or treatment as usual control groups (except one study using psychological placebo), which are expected to produce higher pooled ESs, compared to metaanalyses, including active treatments, as well. The subgroup analysis in a previous meta-analysis, comparing studies with various control types, confirms this assumption. ${ }^{16}$

Regarding the credibility of our findings, one should consider that although meta-analyses can provide strong empirical evidence, their credibility depends on the original studies being meta-analyzed. In the present metaanalysis, two other issues need to be discussed. First, in the screening process, we excluded six trials, as they had considerable inconsistencies or shortcomings in their reports (e.g., numerous typos in the reported scores). Overall, when $25 \%$ of trials need to be excluded due to major problems, one should consider the possibility that the other included trials also have similar problems that were not identified.

Second, in our previous meta-analysis regarding the efficacy of treatments for chronic pain, when the included 
trials were evaluated altogether, we found considerable problems that might undermine the credibility of our results. For instance, we found that in trials conducted by MA researchers, the SMD was 2.5 times higher than that of trials conducted by $\mathrm{PhD}$ researchers. In the present meta-analysis, although no significant differences were observed, we could not assume that such problems were exclusive to pain intensity.

It seems that psychological interventions can effectively improve the outcomes of Iranian IBS patients. Therefore, it is reasonable to generalize well-established treatment guidelines to the Iranian population to recommend psychotherapies for IBS patients, especially those who have moderate to severe symptoms; those who do not respond well to drugs; and/or those who experience significant psychological distress. ${ }^{11-13,33}$

According to our literature review, some issues need to be considered in future trials. First, the risk of bias was a major concern in our investigated trials, which is easy to address, especially in randomization processes, as it only requires the researcher to be aware of the existence of common pitfalls in these processes and then seek the correct approach (for a guide see Friedman et $\mathrm{al}^{34}$ ). Moreover, these processes should be described in details; it is not sufficient to simply mention that the participants were 'randomized' to the experimental or control groups; rather, one must report how the random sequence was generated and how the sequence was concealed before allocation. Second, we found considerable heterogeneity in the ESs of studies that cannot be explained; therefore, identifying these factors can be a priority in future research.

Third, our investigated trials focused mainly on MDs between the experimental and control groups, which is not of critical importance. On the other hand, we should primarily focus on differences between the groups to determine the percentage of cases experiencing clinically significant improvements ${ }^{35}$ (e.g., a 50 -point reduction in the IBS symptom severity scale is considered a clinically significant improvement). ${ }^{36}$ Overall, practitioners must know if a given treatment can significantly improve the patient's symptoms, while they may not be interested in determining the effect of treatment on the mean score of a group of patients (see Moss-Morris et $\mathrm{al}^{37}$ for an example of RCT, and see Ford et $\mathrm{al}^{14}$ for an example of a metaanalysis).

Fourth, inadequate reporting was another avoidable problem in the trials we investigated. The Consolidated Standards of Reporting Trials (CONSORT) is a comprehensive guideline that ensures adequate reporting of clinical trials. ${ }^{38}$ Also, the Journal of Gastroenterology introduced Rome-IV as a practical tool for designing treatment trials for FGIDs. ${ }^{35}$

The most important limitation of our meta-analysis was the measurement of MD or SMD rather than the response rate as the outcome, which is due to the lack of reports on response rates in almost all included trials. Therefore, we could not estimate the probability of clinical improvement in patients treated with a psychological approach.

In conclusion, the present results suggest that Iranian IBS patients respond well to various types of psychotherapy. These treatments reduce the severity of IBS symptoms, as well as anxiety and depression, and improve the patient's quality of life; however, we could not find sufficient evidence regarding the durability of the results. Overall, the efficacy of psychotherapies may vary for patients, depending on unknown factors, which can be determined in future research.

\section{Authors' Contribution}

GF: Study design, manuscript revision. MF: Study design, manuscript revision. AZ: Study design, data collection, statistical analysis and interpretation, and manuscript writing.

\section{Conflict of Interest Disclosures}

The authors had no conflict of interest to declare.

Ethical Statement

Not applicable.

Acknowledgment

This paper was extracted from a thesis by AZ, submitted to University of Tehran (Farabi Campus).

\section{Supplementary Materials}

Supplementary file 1. Characteristics and Risks of Bias of Included Studies.

\section{Refrences}

1. Drossman DA, Hasler WL. Rome IV-functional GI disorders: disorders of gut-brain interaction. Gastroenterology. 2016;150(6):1257-61. doi: 10.1053/j. gastro.2016.03.035.

2. Lovell RM, Ford AC. Global prevalence of and risk factors for irritable bowel syndrome: a meta-analysis. Clin Gastroenterol Hepatol. 2012;10(7):712-21.e4. doi: 10.1016/j.cgh.2012.02.029.

3. Jahangiri P, Hashemi Jazi MS, Hasanzadeh-Keshteli A, Sadeghpour S, Amini E, Adibi P. Irritable bowel syndrome in Iran: SEPAHAN systematic review No. 1. Int J Prev Med. 2012;3(Suppl 1):S1-9.

4. Hasanzadeh-Keshteli A, Dehestani B, Daghaghzadeh H, Adibi P. Epidemiological features of irritable bowel syndrome and its subtypes among Iranian adults. Ann Gastroenterol. 2015;28(2):253-8.

5. Mönnikes H. Quality of life in patients with irritable bowel syndrome. J Clin Gastroenterol. 2011;45 Suppl:S98-101. doi: 10.1097/MCG.0b013e31821fbf44.

6. Fond G, Loundou A, Hamdani N, Boukouaci W, Dargel A, Oliveira J, et al. Anxiety and depression comorbidities in irritable bowel syndrome (IBS): a systematic review and meta-analysis. Eur Arch Psychiatry Clin Neurosci. 2014;264(8):651-60. doi: 10.1007/s00406-014-0502-z.

7. Roohafza H, Zare Bidaki E, Hasanzadeh-Keshteli A, Daghaghzade H, Afshar H, Adibi P. Anxiety, depression and distress among irritable bowel syndrome and their subtypes: an epidemiological population based study. Adv Biomed Res. 2016;5:183. doi: 10.4103/2277-9175.190938. 
8. Modabbernia MJ, Mansour-Ghanaei F, Imani A, MirsafaMoghaddam SA, Sedigh-Rahimabadi M, YousefiMashhour M, et al. Anxiety-depressive disorders among irritable bowel syndrome patients in Guilan, Iran. BMC Res Notes. 2012;5:112. doi: 10.1186/1756-0500-5-112.

9. Enck P, Aziz Q, Barbara G, Farmer AD, Fukudo S, Mayer EA, et al. Irritable bowel syndrome. Nat Rev Dis Primers. 2016;2:16014. doi: 10.1038/nrdp.2016.14.

10. Van Oudenhove L, Crowell MD, Drossman DA, Halpert $\mathrm{AD}$, Keefer L, Lackner JM, et al. Biopsychosocial aspects of functional gastrointestinal disorders. Gastroenterology. 2016. doi: 10.1053/j.gastro.2016.02.027.

11. National Institute for Health and Care Excellence (NICE). Irritable Bowel Syndrome in Adults: Diagnosis and Management. NICE Clinical Guidelines, No. 61. London: NICE; 2017.

12. Spiller R, Aziz Q, Creed F, Emmanuel A, Houghton L, Hungin P, et al. Guidelines on the irritable bowel syndrome: mechanisms and practical management. Gut. 2007;56(12):1770-98. doi: 10.1136/gut.2007.119446.

13. Khan S, Chang L. Diagnosis and management of IBS. Nat Rev Gastroenterol Hepatol. 2010;7(10):565-81. doi: 10.1038/nrgastro.2010.137.

14. Ford AC, Lacy BE, Harris LA, Quigley EMM, Moayyedi P. Effect of antidepressants and psychological therapies in irritable bowel syndrome: an updated systematic review and meta-analysis. Am J Gastroenterol. 2019;114(1):21-39. doi: 10.1038/s41395-018-0222-5.

15. Schaefert R, Klose P, Moser G, Häuser W. Efficacy, tolerability, and safety of hypnosis in adult irritable bowel syndrome: systematic review and meta-analysis. Psychosom Med. 2014;76(5):389-98. doi: 10.1097/ psy.0000000000000039.

16. Li L, Xiong L, Zhang S, Yu Q, Chen M. Cognitivebehavioral therapy for irritable bowel syndrome: a metaanalysis. J Psychosom Res. 2014;77(1):1-12. doi: 10.1016/j. jpsychores.2014.03.006.

17. Liegl G, Plessen CY, Leitner A, Boeckle M, Pieh C. Guided self-help interventions for irritable bowel syndrome: a systematic review and meta-analysis. Eur J Gastroenterol Hepatol. 2015;27(10):1209-21. doi: 10.1097/ meg.0000000000000428.

18. Francisconi CF, Sperber AD, Fang X, Fukudo S, Gerson MJ, Kang JY, et al. Multicultural aspects in functional gastrointestinal disorders (FGIDs). Gastroenterology. 2016;150(6):1344-54.e2. doi: 10.1053/j.gastro.2016.02.013.

19. Koç V, Kafa G. Cross-cultural research on psychotherapy: the need for a change. J Cross Cult Psychol. 2019;50(1):10015. doi: $10.1177 / 0022022118806577$.

20. Higgins JP, Deeks JJ. Selecting studies and collecting data. In: Higgins JP, Green S, eds. Cochrane Handbook for Systematic Reviews of Interventions: Cochrane Book Series. John Wiley \& Sons Ltd; 2008. p. 151-85. doi: 10.1002/9780470712184.ch7.

21. Higgins JP, Altman DG. Assessing risk of bias in included studies. In: Higgins JP, Green S, eds. Cochrane Handbook for Systematic Reviews of Interventions. Chichester, UK: John Wiley \& Sons Ltd; 2008. p. 187-241. doi: 10.1002/9780470712184.ch8.

22. Morris SB. Estimating effect sizes from pretest-posttestcontrol group designs. Organ Res Methods. 2008;11(2):36486. doi: 10.1177/1094428106291059.
23. Higgins JP, Deeks JJ, Altman DG. Special topics in statistics. In: Higgins JP, Green S, eds. Cochrane Handbook for Systematic Reviews of Interventions: Cochrane Book Series. John Wiley \& Sons Ltd; 2008. p. 481-529. doi: 10.1002/9780470712184.ch16.

24. Solati-Dehkordi K. A Comperhensive Study of CognitiveBehavioral Therapy, Life Style Training, Relaxation and Medication Therapies on Quality of Life, Psychological Symptoms, Severity and Frequency of Symptoms IBS-d Patients [thesis]. Isfahan: University of Isfahan; 2008.

25. Langan D, Higgins JP, Jackson D, Bowden J, Veroniki AA, Kontopantelis E, et al. A comparison of heterogeneity variance estimators in simulated random-effects metaanalyses. Res Synth Methods. 2019;10(1):83-98. doi: 10.1002/jrsm.1316.

26. Deeks JJ, Higgins JP, Altman DG. Analysing data and undertaking meta-analyses. In: Higgins JP, Green S, eds. Cochrane Handbook for Systematic Reviews of Interventions: Cochrane Book Series. John Wiley \& Sons Ltd; 2008. p. 243-96. doi: 10.1002/9780470712184.ch9.

27. Zia-Tohidi A, Jandaghi G, Firoozi M. Meta-analysis of psychological intervention for chronic pain: challenges and strategies for research quality improvement. Journal of Anesthesiology and Pain. 2019;10(3):91-104. [Persian].

28. Sterne JA, Egger M, Moher D. Addressing reporting biases. In: Higgins JP, Green S, eds. Cochrane Handbook for Systematic Reviews of Interventions: Cochrane Book Series. John Wiley \& Sons Ltd; 2008. p. 297-333. doi: 10.1002/9780470712184.ch10.

29. Jin ZC, Zhou XH, He J. Statistical methods for dealing with publication bias in meta-analysis. Stat Med. 2015;34(2):34360. doi: 10.1002/sim.6342.

30. Viechtbauer W. Conducting meta-analyses in $\mathrm{R}$ with the metafor package. J Stat Softw. 2010;36(3):1-48. doi: 10.18637/jss.v036.i03.

31. Laird KT, Tanner-Smith EE, Russell AC, Hollon SD, Walker LS. Short-term and long-term efficacy of psychological therapies for irritable bowel syndrome: a systematic review and meta-analysis. Clin Gastroenterol Hepatol. 2016;14(7):937-47.e4. doi: 10.1016/j.cgh.2015.11.020.

32. Wood L, Egger M, Gluud LL, Schulz KF, Jüni P, Altman DG, et al. Empirical evidence of bias in treatment effect estimates in controlled trials with different interventions and outcomes: meta-epidemiological study. BMJ. 2008;336(7644):601-5. doi: 10.1136/bmj.39465.451748.AD.

33. Moayyedi P, Mearin F, Azpiroz F, Andresen V, Barbara G, Corsetti $\mathrm{M}$, et al. Irritable bowel syndrome diagnosis and management: a simplified algorithm for clinical practice. United European Gastroenterol J. 2017;5(6):773-88. doi: 10.1177/2050640617731968.

34. Friedman LM, Furberg CD, DeMets DL, Reboussin DM, Granger CB. Fundamentals of Clinical Trials. 5th ed. Springer; 2016.

35. Irvine EJ, Whitehead WE, Chey WD, Matsueda K, Shaw M, Talley NJ, et al. Design of treatment trials for functional gastrointestinal disorders. Gastroenterology. 2006;130(5):1538-51. doi: 10.1053/j.gastro.2005.11.058.

36. Francis CY, Morris J, Whorwell PJ. The irritable bowel severity scoring system: a simple method of monitoring irritable bowel syndrome and its progress. Aliment Pharmacol Ther. 1997;11(2):395-402. doi: 10.1046/j.13652036.1997.142318000.x. 
37. Moss-Morris R, McAlpine L, Didsbury LP, Spence MJ. A randomized controlled trial of a cognitive behavioural therapy-based self-management intervention for irritable bowel syndrome in primary care. Psychol Med. 2010;40(1):85-94. doi: 10.1017/s0033291709990195.
38. Boutron I, Altman DG, Moher D, Schulz KF, Ravaud P. CONSORT statement for randomized trials of nonpharmacologic treatments: a 2017 update and a CONSORT extension for nonpharmacologic trial abstracts. Ann Intern Med. 2017;167(1):40-7. doi: 10.7326/m17-0046. 